\title{
Analysis of powder-snow avalanches using three-dimensional topographic data
}

\author{
Yusuke Fukushima and Norio Hayakawa \\ Department of Civil Engineering, Nagaoka University of Technology, Nagaoka, Niigata 940-21, Japan
}

\begin{abstract}
A fluid-dynamical model of powder-snow avalanches is developed which takes into account three-dimensional topography and can compute an avalanche's running course. The model also predicts the variations of height, speed and concentration of snow particles in the avalanche, as well as the level of turbulence. Application of the model to the Maseguchi avalanche, which occurred in 1986 at Niigata, Japan, shows it can provide reasonable prediction of the running course, height and speed of an avalanche.
\end{abstract}

\section{INTRODUCTION}

Powder-snow avalanches occur when some external force such as the wind initially acts to suspend non-cohesive powder snow on a steep mountain slope. The snow particles suspended by the turbulent motion of air are then impelled by gravity toward the steepest direction, thus leading to the formation of a powder-snow avalanche. The powder-snow avalanche rapidly grows as increasing quantity of snow particles is suspended from the dry-snow layer below. Thus, the entrainment of dry snow particles from the bed is essential to avalanche development. The turbulence of the air-snow mixture itself acts to create new turbulence. However, if the intensity of turbulence is not strong enough to keep snow particles in suspension, the avalanche runs out with resulting deposition of the initially suspended snow particle.

Recently, the avalanche dynamic has been a subject of considerable study (Hopfinger, 1983; Hutter and Nohguchi, 1990). Most of these studies, however, are restricted to stream avalanches. It is clear that studies of power-snow avalanches are warranted. Fukushima (1986) and Fukushima and Parker (1990) developed the fluid-dynamical theory of self-accelerating powdersnow avalanches and applied it to the Maseguchi avalanche, which occurred at Maseguchi, Niigata Prefecture, Japan, on 26 January 1986. The results of their numerical simulation of the powder snow avalanche are quite reasonable. The model of Fukushima and Parker (1990) cannot, however, predict the running course of the avalanche, as it is an input to the model. From the engineering point of view, it is desirable that the running course can be estimated by a simulation model based on the known topography of the mountain slope.

In the new model presented here, the running course is simulated along with other dynamical quantities. The model can take into account the three-dimensional topography of mountain slopes. It consists of the two- dimensional treatment of momentum balance, the mass conservation equations of air and snow, and an approximate equation of conservation of turbulent energy.

\section{TOPOGRAPHY OF THE SLOPE AND ITS MODELING}

Generally, the topography of the mountainous areas where avalanches occur is complicated. As a result, some kind of modeling of topography is needed. In our investigation, ground topography is discretized in terms of triangular elements. The positions and the elevations are given at the nodal points of each element. The elevation is taken to vary linearly within a given element, so that the slope is constant. The total coordinates are described by $(X, Y, Z)$ and the local coordinates on the element are described by $(x, y, z)$. The horizontal distance on the $(X, Y)$ plane is $S$ and the local distance in the inclined plane is $s$. The vector normal to the slope is expressed by $\mathbf{i}_{\mathbf{n}}$ and its components are defined by $\left(i_{\mathrm{n} x}, i_{\mathrm{n} y}, i_{\mathrm{n} z}\right)$.

The direction angle of the steepest slope $\phi$ and the slope angle $\theta_{0}$, as shown in Figure 1 , are related to the normal vector as follows:

$$
\phi=\tan ^{-1}\left(\left|i_{\mathrm{n} y} / i_{\mathrm{n} x}\right|\right)
$$

and

$$
\theta_{0}=\cos ^{-1}\left(i_{\mathrm{n} z} /\left|\mathbf{i}_{\mathbf{n}}\right|\right) .
$$

The angles of inclination in the $x$ and $y$ directions are obtained from $\phi$ and $\theta_{0}$, as shown in Figure 1, as follows:

$$
\theta_{x}=\tan ^{-1}\left(\frac{\sin \theta_{0} \cos \phi}{\cos \theta_{0}}\right)=\tan ^{-1}\left(\tan \theta_{0} \cos \phi\right)
$$

and

$$
\theta_{y}=\tan ^{-1}\left(\frac{\sin \theta_{0} \sin \phi}{\cos \theta_{0}}\right)=\tan ^{-1}\left(\tan \theta_{0} \sin \phi\right) .
$$




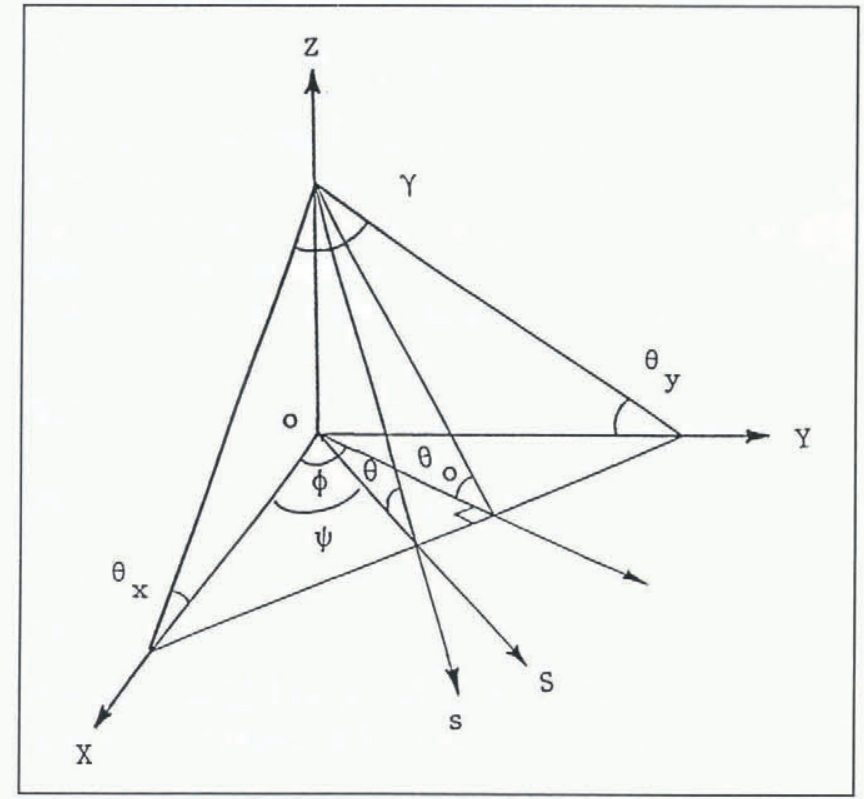

Fig. 1. Angles of directions of the maximum downward slope and running course of the avalanche.

The direction of the running course and the direction of the steepest slopes are not generally identical. As shown in Figure 1, the direction angle of the running course defined relative to the $x$ axis is denoted by $\psi$. The slope angle $\theta$ along the running course can thus be expressed in terms of the geometric relation between the direction angle of the avalanche $\psi$, the direction angle of the steepest slope $\phi$, and the angle of the steepest slope $\theta_{0}$ and the angle $\gamma$ as follows:

$$
\theta=\tan ^{-1}\left(\cos (\phi-\psi) \tan \theta_{0}\right)
$$

and

$$
\gamma=\cos ^{-1}\left(\sin \theta_{x} \sin \theta_{y}\right) .
$$

Insofar as the three-dimensional topographical data is given, the direction of the steepest slope $\theta_{0}$ and the maximum slope angle $\phi$ can be obtained from Equations (1) and (2). The slope angles in the $x$ and $y$ directions, as well as $\theta_{x}$ and $\theta_{y}$, are calculated from Equations (3) and (4).

\section{THEORY OF POWDER SNOW AVALANCHES}

\section{Basic equations}

An important characteristic of powder-snow avalanches is that the total buoyancy of the avalanche varies with time and location via erosion or deposition of the surface snow layer. The modeled shape of a powder-snow avalanche is shown in Figure 2 in which $\rho$ and $\rho_{\mathrm{a}}$ are the densities of the avalanche and the air, respectively. $P_{\mathrm{b}}$ and $P_{\mathrm{i}}$ are the lengths of the bottom and the interface between air and the avalanche, respectively. $\theta$ is the slope angle, $h$ is the height, $t$ is time, $A$ is the area of the avalanche in the transverse direction, $C$ is the mean volume concentration of snow, and $K$ is the mean turbulent intensity in the avalanche. $U$ and $V$ are velocity components in the $x$ and $y$ directions, respectively, and $U_{\text {abs }}$ is the absolute velocity of the avalanche. It is assumed that the shape of the avalanche is uniform in the transverse direction to the flow. The basic equations of motion are the mass conservation equations of air and snow, the momentum equations in the $x$ and $y$ directions, and an approximate equation of conservation of turbulent kinetic energy (Fukushima, 1986). The mass conservation equation for air may be written as

$$
\frac{\mathrm{d} A}{\mathrm{~d} t}=E_{\mathrm{a}} U_{\mathrm{abs}} P_{\mathrm{i}} .
$$

The mass conservation equation of snow particles is expected to be

$$
\frac{\mathrm{d}}{\mathrm{d} t} C A=v_{\mathrm{s}}\left(E_{\mathrm{s}}-c_{\mathrm{b}} \cos \theta\right) P_{\mathrm{b}} .
$$

The momentum equation in the $x$-direction takes the form

$$
\begin{aligned}
\frac{\mathrm{d}}{\mathrm{d} t}\left(\rho+k_{\mathrm{v}} \rho_{\mathrm{a}}\right) U A= & \left(\rho-\rho_{\mathrm{a}}\right) g A \sin \theta_{x} \\
& -\left(\tau_{\mathrm{i}} P_{\mathrm{i}}+\tau_{\mathrm{b}} P_{\mathrm{b}}\right) U / U_{\mathrm{abs}} .
\end{aligned}
$$

The momentum equation in the $y$ direction takes the form

$$
\begin{aligned}
\frac{\mathrm{d}}{\mathrm{d} t}\left(\rho+k_{\mathrm{v}} \rho_{\mathrm{a}}\right) V A= & \left(\rho-\rho_{\mathrm{a}}\right) g A \sin \theta_{y} \\
& -\left(\tau_{\mathrm{i}} P_{\mathrm{i}}+\tau_{\mathrm{b}} P_{\mathrm{b}}\right) V / U_{\text {abs }} .
\end{aligned}
$$

The equation of the level of turbulence can be expressed as

$$
\begin{aligned}
\frac{\mathrm{d}}{\mathrm{d} t}\left(\rho+k_{\mathrm{v}} \rho_{\mathrm{a}}\right) K A= & \left(\tau_{\mathrm{i}} P_{\mathrm{i}}+\tau_{\mathrm{b}} P_{\mathrm{b}}\right) U_{\mathrm{abs}} \\
& +\frac{1}{2} \rho_{\mathrm{a}}\left\{\left(1+k_{\mathrm{v}}\right) E_{\mathrm{a}} U_{\mathrm{abs}} P_{\mathrm{i}}\right. \\
& \left.+R v_{\mathrm{s}}\left(E_{\mathrm{s}}-c_{\mathrm{b}} \cos \theta\right) P_{\mathrm{b}}\right\} U_{\mathrm{abs}}^{2} \\
& -\rho_{\mathrm{a}} R g v_{\mathrm{s}} A-\beta \rho K^{\frac{3}{2}} A / h \\
& -\xi_{\mathrm{p}} R g \cos \theta h\left\{(1 / 2) C E_{\mathrm{a}} U_{\mathrm{abs}} P_{\mathrm{i}}\right. \\
& \left.+v_{\mathrm{s}}\left(E_{\mathrm{s}}-c_{\mathrm{b}} \cos \theta\right) P_{\mathrm{b}}\right\} .
\end{aligned}
$$

The absolute avalanche speed, $U_{\text {abs }}$, is described as

$$
U_{\mathrm{abs}}=\sqrt{(U+V \cos \gamma)^{2}+(V \sin \gamma)^{2}}
$$

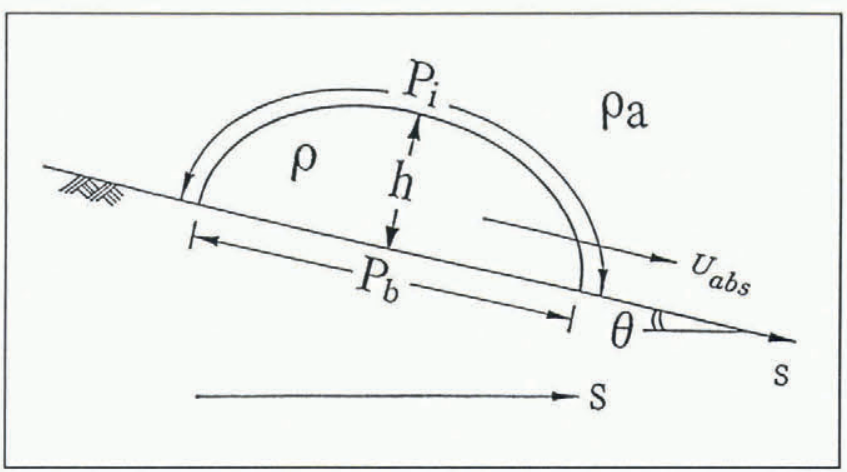

Fig. 2. Schematic view of a modeled powder-snow avalanche. 
In Equations (7)-(11), $v_{8}$ is the terminal velocity of snow particles in air and $k_{\mathrm{v}}$ is the added mass coefficient, $\beta$ is a non-dimensional coefficient related to the viscous dissipation rate of turbulent energy in the avalanche, and $\xi_{\mathrm{A}}, \xi_{\mathrm{t}}$, $\xi_{\mathrm{b}}$, and $\xi_{\mathrm{p}}$ are shape factors (described below). $E_{\mathrm{a}}$ and $E_{\mathrm{s}}$ are coefficients describing the entrainment of air from above and snow particles from below. The location of the avalanche is obtained from the integration of the following two equations:

$$
\frac{\mathrm{d} x}{\mathrm{~d} t}=U \cos \theta_{x}
$$

and

$$
\frac{\mathrm{d} x}{\mathrm{~d} t}=U \cos \theta_{y} .
$$

Here $x$ and $y$ are the local coordinates in an element. The direction of the running course is calculated by the relation

$$
\phi=\tan ^{-1}\left(\frac{U \cos \theta_{x}}{V \cos \theta_{y}}\right) .
$$

In order to solve Equations (7)-(14), it is necessary to specify several constitutive relations. The shear stresses acting on the bottom $\tau_{\mathrm{b}}$ and the interface $\tau_{\mathrm{i}}$ are taken to obey the following respective relationships:

$$
\tau_{\mathrm{i}}=\rho_{\mathrm{a}} \alpha K
$$

and

$$
\tau_{\mathrm{b}}=\rho_{\mathrm{b}} \alpha K
$$

Here, $\alpha$ is a non-dimensional coefficient which must be determined with reference to experimental data, $\rho_{\mathrm{b}}$ the mean bottom density of avalanche, and $\rho_{\mathrm{a}}$ the density of air. These are given in terms of the following relations where $\rho$ denotes the overall density of avalanche:

$$
\rho=\rho_{\mathrm{a}}(1+R C)
$$

and

$$
\rho_{\mathrm{b}}=\rho_{\mathrm{a}}\left(1+R c_{\mathrm{b}}\right) .
$$

Hence, $c_{\mathrm{b}}$ is the near-bottom volume concentration of snow in the avalanche, and $R=\left(\rho-\rho_{\mathrm{a}}\right) / \rho_{\mathrm{a}}$ is the effective specific gravity of snow immersed in air.

The entrainment coefficients of air and snow, $E_{\mathrm{a}}$ and $E_{8}$, are expressed by the following relations, respectively (Escudier and Maxworthy, 1973; Beghin and others, 1981; Akiyama and Fukushima, 1984):

$$
E_{\mathrm{a}}=0.1\left(\theta / 90^{\circ}\right) \text {, }
$$

where $\theta$ is measured in degrees $\left({ }^{\circ}\right)$, and

$$
E_{\mathrm{s}}=\left(3.0 \times 10^{-12}\right) Z^{10}\left(1-Z_{\mathrm{c}} / Z\right),
$$

for $Z_{\mathrm{c}}<Z<Z_{\mathrm{m}}$. For $Z>Z_{\mathrm{m}}, E_{\mathrm{s}}=0.3$; and $E_{\mathrm{s}}=0$ for $Z<Z_{\mathrm{c}}$, in which the lower limit of $Z, Z_{\mathrm{c}}=5$, and the maximum limit of $Z, Z_{\mathrm{m}}=13.2$. In Equation (21), $Z=\mu \sqrt{R_{\mathrm{p}}}, \mu=\left(1 / \nu_{\mathrm{s}}\right) \sqrt{\alpha K}$, and $R_{\mathrm{p}}=\left(D_{\mathrm{s}} / \nu\right) \sqrt{R g D_{\mathrm{s}}}$, the last being the particle Reynolds number, where $D_{\mathbf{B}}$ denotes the characteristic diameter of the snow particles, $\nu$ is the kinetic viscosity of air, and $\mu$ is the nondimensional shear velocity. Equation (22) indicates the relation between mean and bottom concentration in the avalanche:

$$
\begin{aligned}
r_{0} & =c_{\mathrm{b}} / C \\
& =1+31.5 \mu^{-1.46}
\end{aligned}
$$

The above relation is obtained based on the analogy to the concentration distribution for open-channel suspensions flow expressed by the Rouse equation. Equation (22) was introduced by Parker (1982) in order to simulate turbidity currents in the ocean. The shape of the avalanche is described by

$$
\begin{aligned}
& A=\xi_{\mathrm{A}} h^{2}, \\
& P_{\mathrm{b}}=\xi_{\mathrm{b}} h,
\end{aligned}
$$

and

$$
P_{\mathrm{i}}=\xi_{\mathrm{i}} h,
$$

where the shape factors $\xi_{\mathrm{p}}, \xi_{\mathrm{A}}, \xi_{\mathrm{b}}$ and $\xi_{\mathrm{i}}$ are expressed below assuming that the shape of an avalanche is half an ellipse:

$$
\begin{gathered}
\xi=(\pi / 4) \xi_{\mathrm{b}}, \\
\xi_{\mathrm{i}}=(\pi /(2 \sqrt{2})) \sqrt{\left(4 \xi_{\mathrm{b}}^{-1}+1\right)} \xi_{\mathrm{b}} \\
k_{\mathrm{v}}=2 / \xi_{\mathrm{b}}, \\
\xi_{\mathrm{P}}=4 /(3 \pi), \\
\xi_{\mathrm{b}}=8.47 \theta^{-1 / 3} .
\end{gathered}
$$

Equation (30) is estimated from the experimental results of Beghin and others (1981), as analyzed by the present authors.

The rate of lowering of the elevation of the surface snow layer $\eta_{\mathrm{b}}$, is expressed by a form of the Exner relation for sediment continuity (Fukushima and Parker, 1990) as

$$
\eta_{\mathrm{b}}=\frac{1}{1-\lambda} v_{\mathrm{s}}\left(E_{\mathrm{s}}-c_{\mathrm{b}} \cos \theta\right)
$$

where $\lambda$ is the porosity of the snow layer.

Finally, the functional relationship for $\beta$ as determined by Fukushima (1986) and Fukushima and Parker (1990) takes the form

$$
\begin{aligned}
\beta= & \frac{1}{\xi_{\mathrm{A}}}\left(\frac{\alpha}{c_{\mathrm{D}}}\right)^{3 / 2}\left\{\left(\xi_{\mathrm{i}}+\xi_{\mathrm{b}}\right) c_{\mathrm{D}}+\left(1-\frac{c_{\mathrm{D}}}{2 \alpha}\right)\left(1+k_{\mathrm{v}}\right) \xi_{\mathrm{i}} E_{\mathrm{a}}\right. \\
& \left.-\xi_{\mathrm{P}} \cot \theta \frac{\xi_{\mathrm{i}} E_{\mathrm{a}}}{2 \xi_{\mathrm{A}}}\left[\frac{3}{4}\left(1+k_{\mathrm{v}}\right) \xi_{\mathrm{i}} E_{\mathrm{a}}+\left(\xi_{\mathrm{i}}+\xi_{\mathrm{b}}\right) c_{\mathrm{D}}\right]\right\} .
\end{aligned}
$$

Here, $c_{D}$ is the friction coefficient which must be determined by reference to data. In Equation (32) the various shape factors are functions of slope angle. It thus follows that the coefficient $\beta$ is also a function of the slope angle.

\section{Methodology of the numerical simulation}

In order to simulate a powder-snow avalanche, the topographic domain of interest is discretized in terms of a triangular mesh. All points on the mesh are given threedimensional topographical data, such as the elevation of 
the nodal point. In the model, the geometrical location of the mass center of each triangular element is stored in array form. The simulation is carried out by the integration of Equations (7)-(14) with the aid of Equations (12)-(32). As mentioned earlier, the shape of the avalanche varies with slope angle; thus the volume of the avalanche continuously varies. The unknown variables are $A, U, V, C$ and $K$, and the height of the avalanche is calculated from Equation (23).

\section{APPLICATION TO MASEGUCHI AVALANCHE}

The properties of snow particles are known to vary with meteorological conditions. We assume that the diameter of the snow particles $D_{\mathrm{s}}=0.15 \mathrm{~mm}$, and the effective specific gravity of snow $R=710$ (same value as for pure ice). The corresponding fall velocity in air $v_{8}=0.45 \mathrm{~m} \mathrm{~s}^{-1}$. The initial height of the avalanche $h_{0}=10 \mathrm{~m}$. The initial area $A_{0}$ is calculated by Equation (23). The drag coefficient $c_{\mathrm{D}}$ and the non-dimensional coefficient $\alpha$ are taken to be 0.02 and 0.1 , respectively. The initial values of the speed of the avalanche and the concentration are given by the condition that the avalanche just barely begins to self-accelerate.

Figure 3 shows the running course of the avalanche as simulated by the present model. Three locations are chosen as starting points for avalanches. The avalanche starting from the southeast slope of Mount Gongen is seen to turn strongly to the left as it runs out. The avalanches starting from the two eastern points tend to curve to the

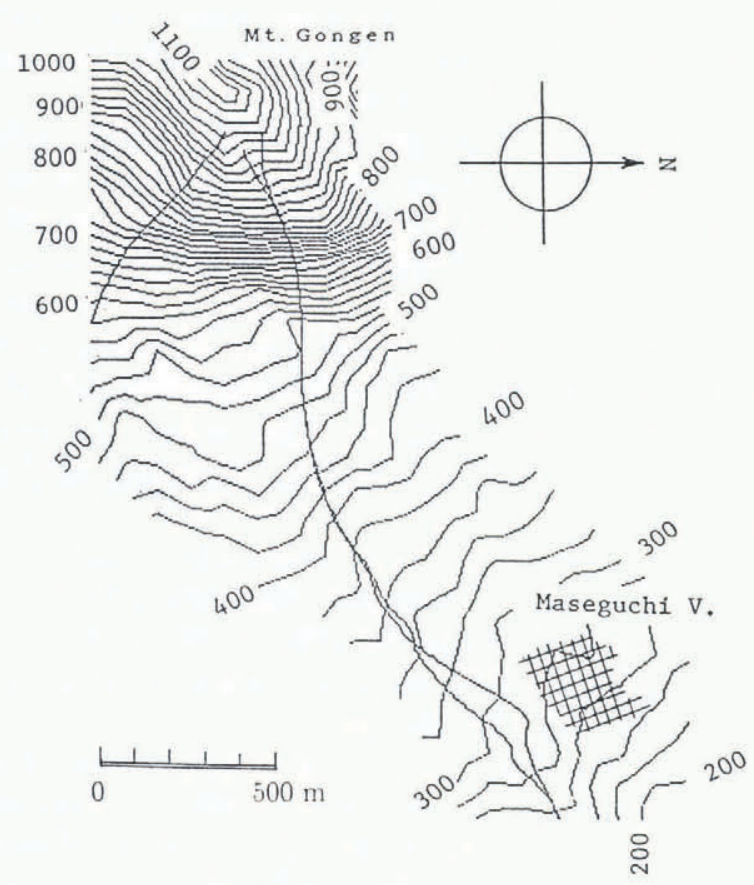

Fig. 3. Running course of the avalanche simulated by the present model.

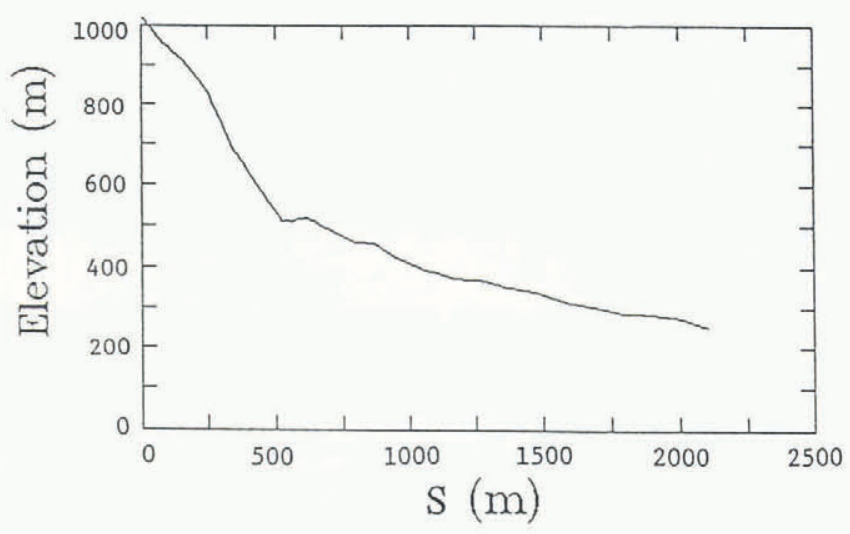

Fig. 4. Elevation along the running course of the avalanche. The abscissa corresponds to the horizontal distance along the running course.

right, thus approaching Maseguchi village. These running courses are very close to the one observed by Fukushima and Parker (1990) and Kobayashi and Izumi, (1988). They indicated an avalanche width of $200 \mathrm{~m}$. The results of the present simulation indicate that the avalanche reaches Maseguchi village. The model thus predicts the disaster of 1986 in which 11 houses were destroyed and 13 people were killed.

The results of the numerical simulation of an avalanche reaching Maseguchi are shown in Figures 4 8. Figure 4 indicates the variation of the elevation along the running course of an avalanche in which the abscissa is the horizontal distance of the running course. As shown

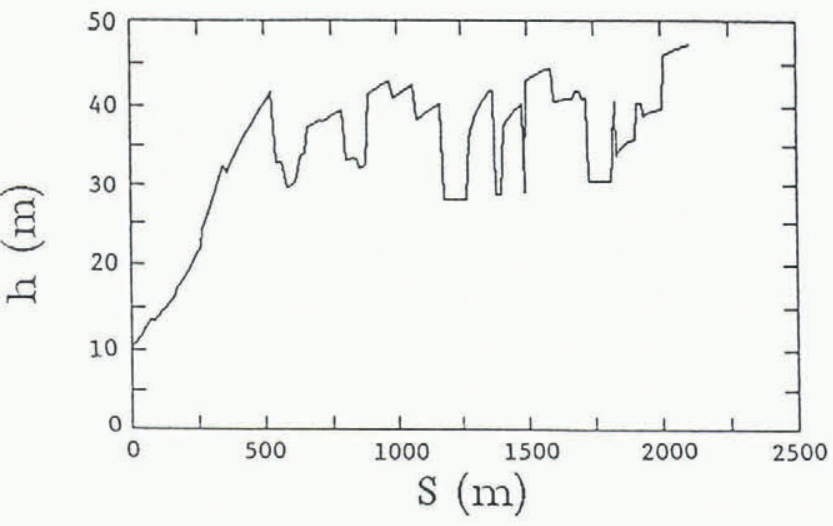

Fig. 5. Variation of the height of the avalanche. The abscissa is the same as that of Fig. 4.

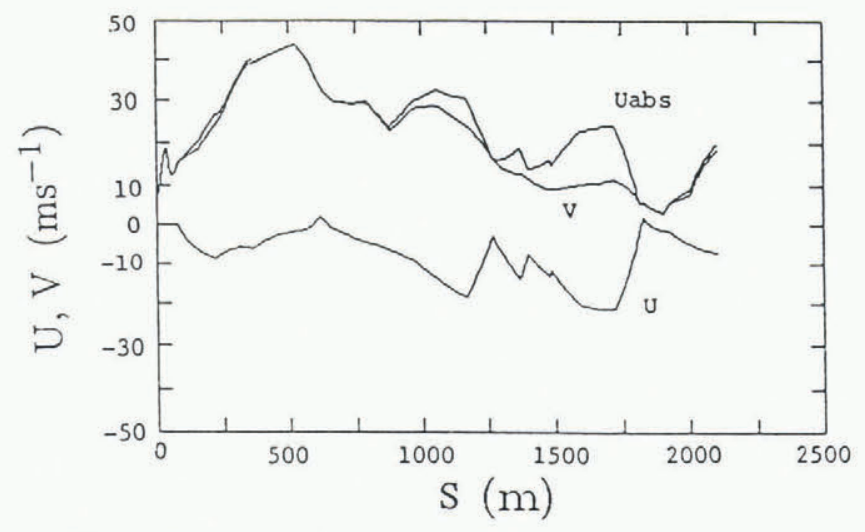

Fig. 6. Variation of the speed of the avalanche. 


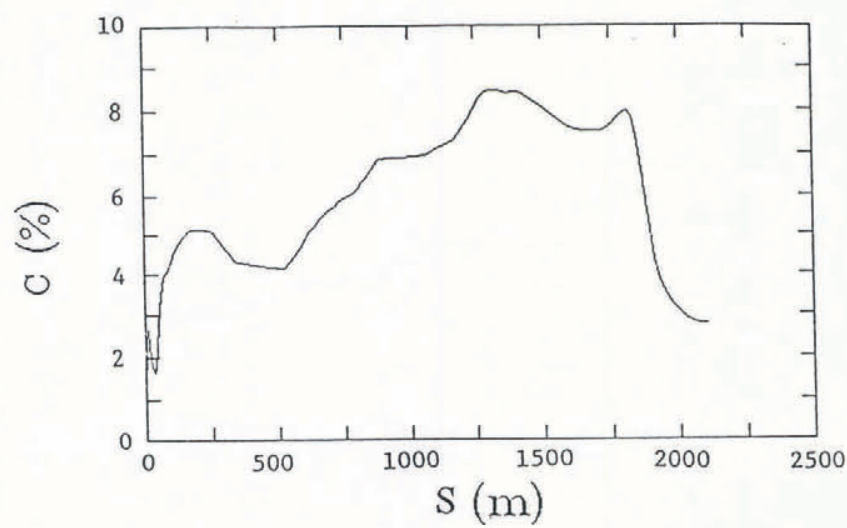

Fig. 7. Variation of the volume concentration of snow in the avalanche.

in Figure 5, the height of the avalanche increases until the avalanche has moved $600 \mathrm{~m}$ downslope of its starting point. Subsequently the avalanche height remains constant. As the avalanche reaches from 0 to $600 \mathrm{~m}$, the slope is quite large (Fig. 4) and the entrainment of air (Equation (20)) becomes large. Thus, the rapid increase of the height occurs. Figure 6 indicates that the speed of the avalanche is continuously changing dependent on the local slope angle. These results are nearly identical to those of the previous model (of Fukushima and Parker). The volume concentration of snow particles rapidly increases on the steep slope of the east side of Mount Gongen, and then varies mildly with the slope angle as shown in Figure 7. Figure 8 indicates that the level of turbulence, $K /\left(0.5 U_{\text {abs }}^{2}\right)$, varies sensibly depending upon the slope angle. As $U_{\text {abs }}$ decreases quickly at about $S=1700 \mathrm{~m}$, the level of turbulence increases rapidly.

The advantage of the present model over the previous model is that an estimated running course of the avalanche can be computed using three-dimensional topographical data. Some problems, however, still remain. For example, the present model is assumed to be two-dimensional in the transverse direction, and the snow entrainment coefficient $E_{8}$ is only crudely estimated based on an analogy to open-channel suspensions. We are presently conducting experiments on three-dimensional thermals in order to understand further the dynamic behavior of three-dimensional powder-snow avalanches. The results of the latter experimental data should help develop a theory of three-dimensional powder-snow avalanches.

\section{CONGLUSIONS}

A theory of self-reinforcing powder-snow avalanches which takes into account arbitrary three-dimensional surface topography has been developed. The theory is applied to the Maseguchi avalanche which occurred in 1986, in Niigata, Japan. The advantage of the present model is that the running course can be computed using three-dimensional topographical data. The present avalanche theory can also predict the variations of height, speed, volume concentration of snow particles, avalanche volume, and the rate of erosion from or deposition onto the bed surface. The results of the

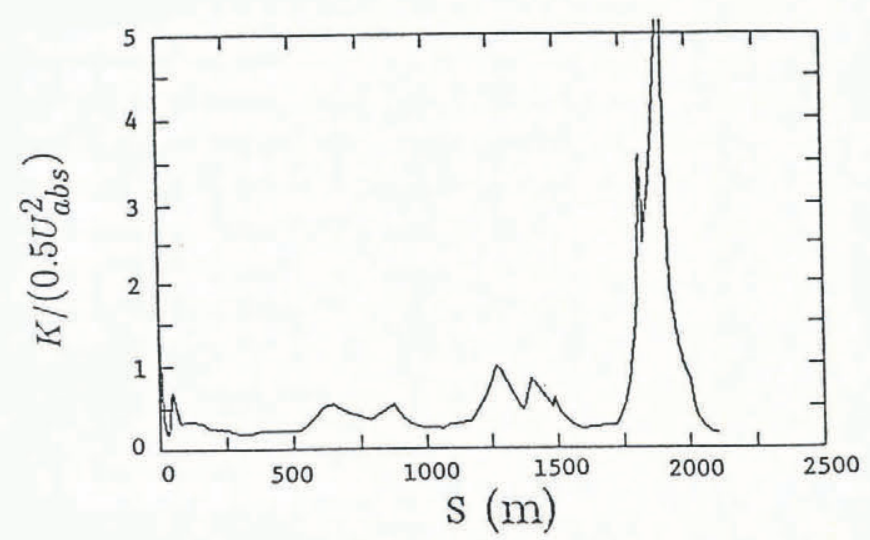

Fig. 8. Variation of the level of turbulence in the avalanche along the flow direction.

numerical simulation of the Maseguchi avalanche are satisfactory.

The present model is essentially two-dimensional in nature. That is, the internal structure of the avalanche is not described. The snow entrainment function represented only a rough estimate based on a loose analogy to the open-channel suspensions. These points should be refined in future.

\section{ACKNOWLEDGEMENT}

Funding from the Japan National Science Foundation (grant no. 04201210) is gratefully acknowledged.

\section{REFERENCES}

Akiyama, J. and Y. Fukushima. 1984. Entrainment of noncohesive bed sediment into suspension. Minneapolis, MN, University of Minnesota. St. Anthony Falls Hydraulic Laboratory. (Memorandum 195.)

Beghin, P., E.J. Hopfinger and R.E. Britter. 1981. Gravitational convection from instantaneous sources on inclined boundary. 7. Fluid Mech., 107, 407-422.

Escudier, M. P. and T. Maxworthy. 1973. On the motion of turbulent thermals. F. Fluid Mech., 61(3), 541-552.

Fukushima, Y. 1986. Analytical study of powder snow avalanches. Seppyo. F. Jpn Soc. Snow Ice, 48(4), 189-197.

Fukushima, Y. and G. Parker. 1990. Numerical simulation of powder snow avalanches. F. Glaciol., 36(123), 229-237.

Hopfinger, E.J. 1983. Snow avalanche motion and related phenomena. Annu. Rev. Fluid Mech., 15, 47-76.

Hutter, K. and Y. Nohguchi. 1990. Similarity solutions for a Voellmy model of snow avalanches with finite mass. Acta Mech., 82, 99-127.

Kobayashi, S. and K. Izumi. 1988. A brief review of the Maseguchi avalanche in Japan in 1986. In International Snow Science Workshop, October, 12-15, Whistler. Proceedings. Ottawa, Canadian Avalanche Association, 57-62.

Parker, G. 1982. Conditions for the ignition of catastrophically erosive turbidity currents. Mar. Geol., 46, 307-327.

The accuracy of references in the text and in this list is the responsibility of the authors, to whom queries should be addressed. 